\title{
Correction to: Conservative and Semiconservative Random Walks: Recurrence and Transience
}

\section{Vyacheslav M. Abramov ${ }^{1,2}$}

Published online: 19 November 2018

(c) Springer Science+Business Media, LLC, part of Springer Nature 2018

\section{Correction to: J Theor Probab (2018) 31:1900-1922 https://doi.org/10.1007/s10959-017-0747-3}

The aim of this note is to correct the errors in the formulation and proof of Lemma 4.1 in [1] and some claims that are based on that lemma. The correct formulation of the aforementioned lemma should be as follows.

Lemma 4.1 Let the birth-and-death rates of a birth-and-death process be $\lambda_{n}$ and $\mu_{n}$ all belonging to $(0, \infty)$. Then, the birth-and-death process is transient if there exist $c>1$ and a value $n_{0}$ such that for all $n>n_{0}$

$$
\frac{\lambda_{n}}{\mu_{n}} \geq 1+\frac{1}{n}+\frac{c}{n \ln n},
$$

and is recurrent if there exists a value $n_{0}$ such that for all $n>n_{0}$

$$
\frac{\lambda_{n}}{\mu_{n}} \leq 1+\frac{1}{n}+\frac{1}{n \ln n} .
$$

Proof Following [2], a birth-and-death process is recurrent if and only if

$$
\sum_{n=1}^{\infty} \prod_{k=1}^{n} \frac{\mu_{k}}{\lambda_{k}}=\infty
$$

The original article can be found online at https://doi.org/10.1007/s10959-017-0747-3.

$\triangle$ Vyacheslav M. Abramov

vabramov126@gmail.com

1 School of Mathematical Sciences, Monash University, Wellington road, Clayton, VIC-3800, Australia

2 School of Science, Royal Melbourne Institute of Technology, GPO Box 2476, Melbourne, VIC-3001, Australia 
Write

$$
\sum_{n=1}^{\infty} \prod_{k=1}^{n} \frac{\mu_{k}}{\lambda_{k}}=\sum_{n=1}^{\infty} \exp \left(\sum_{k=1}^{n} \ln \left(\frac{\mu_{k}}{\lambda_{k}}\right)\right) .
$$

Now, suppose that (1) holds. Then, for sufficiently large $n$

$$
\frac{\mu_{n}}{\lambda_{n}} \leq 1-\frac{1}{n}-\frac{c}{n \ln n}+O\left(\frac{1}{n^{2}}\right)
$$

and since the function $x \mapsto \ln x$ is increasing on $(0, \infty)$, then

$$
\begin{aligned}
\ln \left(\frac{\mu_{n}}{\lambda_{n}}\right) & \leq \ln \left(1-\frac{1}{n}-\frac{c}{n \ln n}+O\left(\frac{1}{n^{2}}\right)\right) \\
& =-\frac{1}{n}-\frac{c}{n \ln n}+O\left(\frac{1}{n^{2}}\right) .
\end{aligned}
$$

Hence, for sufficiently large $n$

$$
\sum_{k=1}^{n} \ln \left(\frac{\mu_{k}}{\lambda_{k}}\right) \leq-\ln n-c \ln \ln n+O(1),
$$

and thus, by (3), for some constant $C<\infty$,

$$
\sum_{n=1}^{\infty} \prod_{k=1}^{n} \frac{\mu_{k}}{\lambda_{k}} \leq C \sum_{n=1}^{\infty} \frac{1}{n(\ln n)^{c}}<\infty
$$

provided that $c>1$. The transience follows.

On the other hand, suppose that (2) holds. Then, for sufficiently large $n$

$$
\frac{\mu_{n}}{\lambda_{n}} \geq 1-\frac{1}{n}-\frac{1}{n \ln n}+O\left(\frac{1}{n^{2}}\right),
$$

and, consequently,

$$
\ln \left(\frac{\mu_{n}}{\lambda_{n}}\right) \geq \ln \left(1-\frac{1}{n}-\frac{1}{n \ln n}+O\left(\frac{1}{n^{2}}\right)\right) .
$$

Similarly to that was provided before, for some constant $C^{\prime}$,

$$
\sum_{n=1}^{\infty} \prod_{k=1}^{n} \frac{\mu_{k}}{\lambda_{k}} \geq C^{\prime} \sum_{n=1}^{\infty} \frac{1}{n \ln n}=\infty
$$

The recurrence follows. 
As $n \rightarrow \infty$, asymptotic expansion (4.5) obtained in the proof of Lemma 4.2 in [1] guarantees its correctness. However, the corrected version of Lemma 4.1 requires more delicate arguments in the proofs of Lemma 4.2 and Theorem 4.13 in [1]. Specifically, in the proof of Lemma 4.2 instead of limit relation (4.6) we should study the cases $d=2$ and $d \geq 3$ separately in terms of the present formulation of Lemma 4.1.

In the formulation of Theorem 4.13 in [1], assumption (4.12) must be replaced by the stronger one:

$$
\frac{L_{n}}{M_{n}} \leq 1+\frac{2-d}{n}+\frac{1-\epsilon}{n \ln n},
$$

for all large $n$ and a small positive $\epsilon$. In the proof of Theorem 4.13 in [1], we should take into account that for large $n$

$$
\frac{\lambda_{n}(1, d)}{\mu_{n}(1, d)}=1+\frac{d-1}{n}+O\left(\frac{1}{n^{2}}\right)
$$

is satisfied (see the proof of Lemma 4.2), and hence,

$$
\frac{p_{n}}{1-p_{n}} \asymp\left[\frac{\lambda_{n}(1, d)}{\mu_{n}(1, d)} \cdot \frac{L_{n}}{M_{n}}\right] \leq 1+\frac{1}{n}+\frac{1-\epsilon}{n \ln n}+\frac{C}{n^{2}},
$$

for a fixed constant $C$ and large $n$. So, according to Lemma 4.1 the process is recurrent.

Note that the statements of Lemma 4.1 are closely related to those of Theorem 3 in [3] that prove recurrence and transience for the model studied there.

Acknowledgements The help of the reviewer is highly appreciated.

\section{References}

1. Abramov, V.M.: Conservative and semiconservative random walks: recurrence and transience. J. Theor. Probab. 31(3), 1900-1922 (2018)

2. Karlin, S., McGregor, J.: The classification of the birth-and-death processes. Trans. Am. Math. Soc. 86(2), 366-400 (1957)

3. Menshikov, M.V., Asymont, I.M., Iasnogorodskii, R.: Markov processes with asymptotically zero drifts. Probl. Inf. Transm. 31, 248-261 (1995), translated from Problemy Peredachi Informatsii 31, 60-75 (in Russian) 